\title{
Panorama situacional da Teleodontologia no mundo: uma revisão integrativa
}

\author{
Cinthia Nara Gadelha Teixeira*; Maria Imaculada de Queiroz Rodrigues**; Luciana Maria Arcanjo

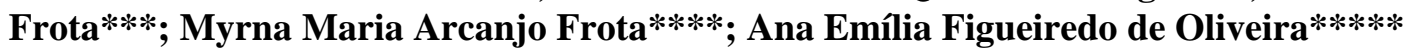

\author{
* Doutoranda, Programa de Pós-Graduação em Odontologia, \\ Universidade Federal do Maranhão \\ ** Estudante, Curso de Odontologia, Universidade Federal do Ceará \\ *** Mestranda, Programa de Pós-Graduação em Clínica \\ Odontológica, Universidade Federal do Ceará \\ **** Doutoranda, Programa de Pós-Graduação em Clínica \\ Odontológica, Docente, Curso de Odontologia, Universidade \\ Federal do Ceará. \\ ***** Professora Associada, Universidade Federal do Maranhão
}

Recebido em 21/06/2017. Aprovado em 09/01/2018.

\begin{abstract}
RESUMO
O uso das Tecnologias de Informação e Comunicação (TIC) para a troca de dados e informações em Odontologia, provendo serviços de saúde em situações em que seja necessário transpor barreiras geográficas, temporais, sociais e culturais é chamado de Teleodontologia. O objetivo dessa revisão integrativa foi analisar o panorama situacional global da Teleodontologia. Foram selecionados artigos científicos publicados nos últimos 10 anos, na íntegra, tanto em português quanto em inglês. A busca dos periódicos foi realizada nas bases de dados Scientific Eletronic Library Online, Literatura Latinoamericana e do Caribe em Ciências da Saúde e PubMed, por meio da combinação dos seguintes descritores "Telemedicine", "Dentistry", "Distance, Education", nas línguas portuguesa e inglesa. Foram encontrados 340 artigos, sendo 14 incluídos para avaliação final, sendo o PubMed a base de dados que mais prevaleceu $(71,4 \%)$. A maioria dos estudos $(85,7 \%)$ foi incluída no nível IV de evidência. Foram identificados estudos informando sobre o uso dessa tecnologia para minimizar as barreiras geográficas e fornecer uma assistência adequada às populações mais vulneráveis e outros sobre relatos de profissionais, estudantes de Odontologia e pacientes quanto ao uso da Teleodontologia. Concluiu-se que a Teleodontologia é uma realidade global, mas ainda necessita de disseminação de suas experiências na comunidade científica, para sua consolidação como uma ferramenta educativa e de assistência à saúde.

Descritores: Tecnologia de Informação. Telemedicina. Informática Odontológica. Odontologia. Educação a Distância.
\end{abstract}




\section{INTRODUÇÃO}

A era da tecnologia provocou mudanças num ritmo frenético, permitido o surgimento de novas alternativas com relação aos recursos educacionais, que trazem entre suas características a flexibilização na entrega de conteúdos educacionais ${ }^{1}$, como a Teleodontologia.

A Teleodontologia como uma nova ferramenta de interação e comunicação foi descrita recentemente, com escopo e significado até então não bem definidos e sem consenso firmado. Ela surge a partir da Telemedicina, sendo definida como o uso das Tecnologias de Informação e Comunicação (TIC) para a troca de dados e informações em saúde e para prover serviços de saúde em situações em que haja necessidade em transpor barreiras geográficas, temporais, sociais e culturais ${ }^{2}$. Nesse cenário, em 2002, a National Health System relatou preocupação com o atendimento odontológico de pacientes que viviam em regiões remotas e isoladas, declarando ser a Teleodontologia uma alternativa de suporte para dentistas que atuavam nessas regiões ${ }^{3}$, apresentando-se também como uma maneira mais acessível de fornecer serviços à população ${ }^{4}$.

No Brasil, o Telessaúde Brasil Redes na Atenção Básica é uma alternativa de melhoria da qualidade do atendimento, a ampliação do escopo de ações ofertadas pelas equipes e o aumento da capacidade clínica, incluindo também serviços de Odontologia. Este é um componente do programa de requalificação das Unidades Básicas de Saúde (UBS), cujo objetivo é ampliar a resolutividade da Atenção Básica, promovendo sua integração com o conjunto da Rede de Atenção à Saúde, a partir do desenvolvimento de ações de apoio à atenção à saúde e de educação permanente para as equipes de Atenção Básica ${ }^{5}$.

Como campo de conhecimento integrante da Telessaúde, a Teleodontologia vem em constante evolução, com ênfase na teleducação interativa, na teleassistência e na produção de pesquisas multicêntricas ${ }^{1,6}$. A teleassistência gera um componente de teleducação. $\mathrm{O}$ profissional qualifica sua ação como cirurgiãodentista (CD), agregando novos conhecimentos de maneira imediata, focado na temática da dúvida/pergunta enviada, permitindo ao $\mathrm{CD}$ contar com auxílio remoto para uma segunda opinião sobre um caso complexo ou dúvidas que porventura venha a ter, também conhecida como segunda opinião formativa ${ }^{7}$. A teleducação também pode colaborar com o profissional de saúde em sua formação profissional ${ }^{8}$, além da formação como residente/especialista, por meio de cursos semipresenciais com suporte remoto ou mesmo telepresenciais, de acordo com a possibilidade ofertada pela especialidade. Tais opções de qualificação possibilitam ao CD novas maneiras de formação, com menor custo, agregando valor e qualidade com melhoria continuada da tomada de decisão clínica ${ }^{9}$.

Assim, a amplitude da ciência oferece espaço para estudo, discussão, e troca de informações para que se construa um caminho adequado e eficaz para a qualificação do profissional $\mathrm{CD}$. Tal atuação poderá refletir positivamente na qualidade de assistência à saúde bucal, possibilitando um melhor planejamento e resultado do tratamento, além de contribuir com o conhecimento multi/ interprofissional. Os conhecimentos específicos da Teleodontologia podem também qualificar a formação/ação da equipe como um todo, integrando um atendimento de qualidade, com respeito às distâncias geográficas e temporais $^{10,11}$.

A Teleodontologia apresenta uma grande variabilidade em seus campos de aplicação e experiências documentadas. Essas experiências podem ser agrupadas em dois ramos principais: 
Teleassistência e Teleeduação $^{12}$. Assim, o objetivo dessa revisão integrativa foi analisar o panorama situacional da Teleodontologia no mundo.

\section{METODOLOGIA}

Trata-se de uma revisão integrativa da literatura, um método de pesquisa utilizado com frequência na prática baseada em evidência, cujo objetivo é reunir e sintetizar resultados anteriores, a fim de elaborar uma explicação abrangente de um fenômeno específico. Assim, as conclusões são estabelecidas mediante a avaliação crítica de diferentes abordagens metodológicas $^{13}$.

Essa revisão integrativa foi composta por cinco etapas: a) elaboração da questão norteadora, a partir da lacuna referente à situação da Teleodontologia no mundo; b) definição das bases de dados e estabelecimento de critérios para inclusão e exclusão de estudos/amostragem ou busca na literatura; c) coleta de dados; análise crítica dos estudos incluídos, seguidos da classificação das evidências dos estudos; d) discussão dos resultados e, por último, e) apresentação da revisão/síntese do conhecimento ${ }^{13}$.

Foram incluídos para compor essa revisão integrativa somente artigos científicos publicados na íntegra, tanto em português quanto em inglês, nos últimos 10 anos (período de 2007 a 2017) e que envolveram os diversos atores e cenários inseridos na Teleodontologia. Aqueles que não estavam condizentes com o tema desse estudo e que estivessem repetidos, foram excluídos.

A busca dos periódicos que compuseram esse trabalho foi realizada nas bases de dados Scientific Eletronic Library Online (SciELO), Literatura Latino-americana e do Caribe em Ciências da Saúde (LILACS) e PubMed, por meio da combinação pelo operador booleano
"AND" dos seguintes descritores, em português e inglês: "Telemedicina/Telemedicine", “Odontologia/Dentistry”, "Educação a Distância/ Distante, Education".

Após as buscas, foi realizada a leitura dos títulos e resumos dos artigos encontrados e excluídos os que não se adequavam ao tema proposto. As buscas realizadas, bem como os artigos encontrados, foram manuseados por um único operador, sendo usado o software gratuito Mendeley para o gerenciamento dos artigos que compuseram essa revisão.

A qualidade dos estudos foi avaliada segundo a classificação do nível de evidência $(\mathrm{NE})^{14}$, da seguinte forma: nível I - evidência obtida do resultado de metanálise de estudos clínicos controlados e com randomização; nível II - evidência obtida em estudo de desenho experimental; nível III - evidência obtida de pesquisas quase-experimentais; nível IV evidências obtidas de estudos descritivos ou com abordagem metodológica qualitativa; nível V evidências obtidas de relatórios de casos ou relatos de experiências; nível VI - evidências baseadas em opiniões de especialistas ou com base em normas ou legislação.

Esse estudo levou em consideração os aspectos éticos da pesquisa, respeitando a autoria das ideias, os conceitos e as definições presentes nos artigos incluídos nessa revisão.

\section{RESULTADOS}

Por meio das combinações dos descritores pré-estabelecidos, foram identificadas 340 publicações, sendo 28 na base de dados SciELO, 115 na LILACS e 197 no PubMed. Depois do emprego dos critérios pré-definidos na metodologia desse estudo, foram selecionados 14 artigos para compor a amostra. A tabela 1 expressa, a partir das estratégias de busca, as bases consultadas, a quantidade de referências encontradas e que foram selecionadas. 
Dos artigos selecionados, $85,7 \%$ tiveram o inglês como idioma de publicação. A maioria dos estudos $(35,7 \%)$ foram publicados em 2011. Os Estados Unidos foram o país mais prevalente da afiliação do principal autor dos artigos $(35,7 \%)$ e a base de dados PubMed prevaleceu $(71,4 \%)$ sobre as demais.

A tabela 2 expressa alguns dados de análise inicial dos artigos selecionados, como autor(es)/ano de publicação, país de afiliação do autor principal, título do artigo e a base de dados onde ele foi encontrado.
Dados que incluem o autor(es)/ano de publicação, objetivos dos estudos, além da evidência dos estudos, segundo o delineamento da pesquisa, encontram-se descritos na tabela 3 .

A maioria dos estudos classificados quanto a evidência no nível IV $(85,7 \%)$, caracterizou evidência de estudos descritivos (não-experimentais), por ser os relatos quanto às experiências sobre o uso da Teleodontologia de cunho observacional, onde descreveram, a partir de observações e análises descritivas, o antes e após das experiências com a implantação dos serviços de Telessaúde ${ }^{15}$.

Tabela 1. Estratégias de busca, bases consultadas, número de referências encontradas e selecionadas

\begin{tabular}{|c|c|c|c|}
\hline Estratégias de busca & $\begin{array}{c}\text { Bases } \\
\text { consultadas }\end{array}$ & $\begin{array}{l}\text { Referências } \\
\text { encontradas }\end{array}$ & $\begin{array}{l}\text { Referências } \\
\text { selecionadas }\end{array}$ \\
\hline \multirow{3}{*}{$\begin{array}{l}\text { Telemedicina/Telemedicine [Palavras] } \\
\text { and Odontologia/ Dentistry [Palavras] }\end{array}$} & SciELO & 03 & 02 \\
\hline & LILACS & 07 & 00 \\
\hline & PubMed & 58 & 09 \\
\hline \multirow{3}{*}{$\begin{array}{l}\text { Telemedicia/Telemedicine [Palavras] } \\
\text { and Educação a Distância/Distance, } \\
\text { Education [Palavras] }\end{array}$} & SciELO & 25 & 01 \\
\hline & LILACS & 80 & 01 \\
\hline & PubMed & 72 & 00 \\
\hline \multirow{3}{*}{$\begin{array}{l}\text { Odontologia/Dentistry [Palavras] and } \\
\text { Educação a Distância/Distance, } \\
\text { Education [Palavras] }\end{array}$} & SciELO & 00 & 00 \\
\hline & LILACS & 23 & 00 \\
\hline & PubMed & 64 & 01 \\
\hline \multirow{3}{*}{$\begin{array}{l}\text { Telemedicina/Telemedicine [Palavras] } \\
\text { and Odontologia/Dentistry [Palavras] } \\
\text { and Educação a distância/Distance, } \\
\text { Education [Palavras] }\end{array}$} & SciELO & 00 & 00 \\
\hline & LILACS & 05 & 00 \\
\hline & PubMed & 03 & 00 \\
\hline \multicolumn{2}{|l|}{ TOTAL } & 340 & 14 \\
\hline
\end{tabular}


Tabela 2. Caracterização dos estudos selecionados

\begin{tabular}{|c|c|c|c|}
\hline Autor/Ano & $\begin{array}{c}\text { País de } \\
\text { afiliação do } \\
\text { autor principal }\end{array}$ & Título do artigo & Base de dados \\
\hline Silva et al., 2011 & Brasil & $\begin{array}{l}\text { Bauru School of Dentistry Tele-Health } \\
\text { League: na educational strategy applied to } \\
\text { research, teaching and extension among } \\
\text { applications in tele-health }\end{array}$ & SciELO \\
\hline $\begin{array}{l}\text { Jampani et al., } \\
2011\end{array}$ & Índia & $\begin{array}{l}\text { Applications of teledentistry: A literature } \\
\text { review and update }\end{array}$ & PubMed \\
\hline $\begin{array}{l}\text { Summerfelt, } \\
2011\end{array}$ & Estados Unidos & $\begin{array}{l}\text { Teledentistry-Assisted, Affiliated } \\
\text { Practice for Dental Hygienists: An } \\
\text { Innovative Oral Health Workforce Model }\end{array}$ & PubMed \\
\hline $\begin{array}{l}\text { Keeppanasserril } \\
\quad \text { et al., } 2011\end{array}$ & Índia & $\begin{array}{l}\text { Effectiveness of Tele-guided Interceptive } \\
\text { Prosthodontic treatment in rural India: A } \\
\text { comparative pilot study }\end{array}$ & PubMed \\
\hline $\begin{array}{l}\text { Costa et al., } \\
\quad 2011\end{array}$ & Brasil & $\begin{array}{l}\text { Teleortodontia: ferramenta de auxílio à } \\
\text { prática clínica e à educação continuada }\end{array}$ & SciELO \\
\hline $\begin{array}{l}\text { Glassman et al., } \\
2012\end{array}$ & Estados Unidos & $\begin{array}{l}\text { The Virtual Dental Home: Implications } \\
\text { for Policy and Strategy }\end{array}$ & PubMed \\
\hline $\begin{array}{l}\text { Skelton-Macedo } \\
\quad \text { et al., } 2012\end{array}$ & Brasil & $\begin{array}{l}\text { Teleodontologia: valores agregados para } \\
\text { o clínico/especialista }\end{array}$ & LILACS \\
\hline $\begin{array}{l}\text { Hilsen, Jones, } \\
2013\end{array}$ & Estados Unidos & $\begin{array}{l}\text { Comparing potential early caries } \\
\text { assessment methods for teledentistry }\end{array}$ & PubMed \\
\hline $\begin{array}{l}\text { Torres-Pereira et } \\
\text { al., } 2013\end{array}$ & Brasil & $\begin{array}{l}\text { Teledentistry: Distant Diagnosis of Oral } \\
\text { Disease Using E-Mails }\end{array}$ & PubMed \\
\hline $\begin{array}{l}\text { Dunbar et al., } \\
2014\end{array}$ & Reino Unido & $\begin{array}{l}\text { The Influence of Using Digital Diagnostic } \\
\text { Information on Orthodontic Treatment } \\
\text { Planning - A Pilot Study }\end{array}$ & PubMed \\
\hline $\begin{array}{l}\text { Kunin et al., } \\
\quad 2014\end{array}$ & Estados Unidos & $\begin{array}{l}\text { Comparing Face-to-Face, Synchronous, } \\
\text { and Asynchronous Learning: } \\
\text { Postgraduate Dental Resident Preferences }\end{array}$ & PubMed \\
\hline $\begin{array}{l}\text { Fortuin, Naidoo, } \\
2015\end{array}$ & África do Sul & $\begin{array}{l}\text { Opportunities for Teledentistry in South } \\
\text { Africa }\end{array}$ & SciELO \\
\hline $\begin{array}{l}\text { Boringi et al., } \\
\quad 2015\end{array}$ & Índia & $\begin{array}{l}\text { Knowledge and Awareness of } \\
\text { Teledentistry among Dental Professionals } \\
\text { - A Cross Sectional Study }\end{array}$ & PubMed \\
\hline $\begin{array}{l}\text { Tesfalul et al., } \\
\quad 2016\end{array}$ & Estados Unidos & $\begin{array}{l}\text { Evaluating the potential impact of a } \\
\text { mobile telemedicine system on } \\
\text { coordination of specialty care for patients } \\
\text { with complicated oral lesions in } \\
\text { Botswana }\end{array}$ & PubMed \\
\hline
\end{tabular}


Tabela 3. Autor(es), ano de publicação, objetivos e nível de evidência dos estudos selecionados

\begin{tabular}{|c|c|c|}
\hline Autor, Ano & Objetivos dos estudos & $\begin{array}{l}\text { Nível de } \\
\text { evidência }\end{array}$ \\
\hline Silva et al., 2011 & $\begin{array}{l}\text { Apresentar as características da Liga Tele-Saúde da FOBUSP, } \\
\text { bem como o desenvolvimento de seus projetos. }\end{array}$ & Nível IV \\
\hline $\begin{array}{l}\text { Jampani et al., } \\
\quad 2011\end{array}$ & $\begin{array}{l}\text { Revisar a origem, racionalidade, escopo, base e requisitos para } \\
\text { Teleodontologia, juntamente com a evidência atual que existe } \\
\text { na literatura, além das questões éticas e legais relacionadas à } \\
\text { prática da Teleodontologia e o futuro deste método alternativo e } \\
\text { inovador de cuidado odontológico. }\end{array}$ & Nível IV \\
\hline $\begin{array}{l}\text { Summerfelt, } \\
2011\end{array}$ & $\begin{array}{l}\text { Fornecer informações sobre a Lei de Proteção ao Paciente e } \\
\text { Assistência Econômica, identifica o equipamento usado no } \\
\text { modelo de Teleodontologia da Universidade do Norte do } \\
\text { Arizona. }\end{array}$ & Nível IV \\
\hline $\begin{array}{l}\text { Keeppanasserril } \\
\quad \text { et al., } 2011\end{array}$ & $\begin{array}{l}\text { Descobrir se os dentistas recém-formados, sob orientação } \\
\text { remota de especialistas, podem fabricar dentaduras que são } \\
\text { funcionais e melhorar a qualidade de vida relacionada à saúde } \\
\text { bucal. }\end{array}$ & Nível III \\
\hline $\begin{array}{l}\text { Costa et al., } \\
\quad 2011\end{array}$ & $\begin{array}{l}\text { Investigar, através de revisão não sistemática da literatura - } \\
\text { quais dentre as tecnologias disponíveis são passíveis de serem } \\
\text { utilizadas no desenvolvimento de serviços de Teleortodontia, } \\
\text { como ferramentas de auxílio à prática clínica e à educação } \\
\text { continuada. }\end{array}$ & Nível IV \\
\hline $\begin{array}{l}\text { Glassman et al., } \\
2012\end{array}$ & Descrever como se dá a Casa Dental Virtual. & Nível IV \\
\hline $\begin{array}{l}\text { Skelton-Macedo } \\
\quad \text { et al., } 2012\end{array}$ & $\begin{array}{l}\text { Retratar, para o clínico/especialista, os valores agregados da } \\
\text { Teleodontologia. }\end{array}$ & Nível IV \\
\hline $\begin{array}{l}\text { Hilsen, Jones, } \\
\quad 2013\end{array}$ & $\begin{array}{l}\text { Avaliar e comparar os métodos de detecção visíveis e de } \\
\text { infravermelho próximo para a identificação da desmineralização } \\
\text { oclusal precoce não cavitada ex vivo. }\end{array}$ & Nível III \\
\hline $\begin{array}{l}\text { Torres-Pereira et } \\
\text { al., } 2013\end{array}$ & $\begin{array}{l}\text { Avaliar a aplicabilidade do telediagnóstico em medicina oral, } \\
\text { através da transmissão de imagens clínicas digitais por e-mail. }\end{array}$ & Nível IV \\
\hline $\begin{array}{l}\text { Dunbar et al., } \\
\quad 2014\end{array}$ & $\begin{array}{l}\text { Avaliar se o planejamento do tratamento ortodôntico é } \\
\text { reproduzível quando realizado usando registros digitais } \\
\text { comparados com exames clínicos ou usando registros } \\
\text { padronizados. }\end{array}$ & Nível IV \\
\hline $\begin{array}{l}\text { Kunin et al., } \\
\qquad 2014\end{array}$ & $\begin{array}{l}\text { Avaliar se o novo formato assíncrono satisfez as necessidades } \\
\text { educacionais dos residentes em comparação com os formatos } \\
\text { tradicional de aula (face a face) e síncrono (ensino à distância). }\end{array}$ & Nível IV \\
\hline $\begin{array}{l}\text { Fortuin, Naidoo, } \\
\quad 2015\end{array}$ & Relatar as oportunidades da Teleodontologia na África do Sul & Nível IV \\
\hline $\begin{array}{l}\text { Boringi et al., } \\
\quad 2015\end{array}$ & $\begin{array}{l}\text { Conhecer o conhecimento e a conscientização da } \\
\text { Teleodontologia entre profissionais dentistas de uma faculdade } \\
\text { de odontologia na Índia. }\end{array}$ & Nível IV \\
\hline $\begin{array}{l}\text { Tesfalul et al., } \\
\quad 2016\end{array}$ & $\begin{array}{l}\text { Explorar o impacto potencial de um sistema de Telemedicina } \\
\text { oral móvel na especialidade de saúde bucal sistema de referência } \\
\text { em Botswana. }\end{array}$ & Nível IV \\
\hline
\end{tabular}


Em um estudo foi revisada a origem, racionalidade, escopo, base e requisitos para Teleodontologia; questões éticas e legais relacionadas à prática da Teleodontologia e o futuro deste método alternativo e inovador de cuidado odontológico. Nele, foi constatado que, diante de todos os desenvolvimentos tecnológicos ocorrendo no campo dessa tecnologia, os profissionais podem eventualmente ligar-se a clínicas odontológicas virtuais e uma era inteiramente nova da Odontologia pode ser criada ${ }^{16}$.

Essa nova era da Odontologia já pode ser observada em um curso de Odontologia da Universidade de São Paulo (USP). O serviço de Teleodontologia da Faculdade de Odontologia de Bauru da USP (TLFOB-USP), em quatro anos de atividade, obteve um alto índice de satisfação e aumento do número de vagas, maior comprometimento dos professores da Universidade e a realização de associação com outras ligas brasileiras. Os membros da TLFOBUSP, ao lado dos professores participantes, desenvolveram projetos em Telessaúde, Teleajuda e Tele-educação, resultando no envolvimento da Universidade e da comunidade $^{17}$.

O uso da Teleodontologia para minimizar as barreiras geográficas e fornecer uma assistência adequada às populações mais vulneráveis foi relatado em algumas das publicações selecionadas por essa revisão ${ }^{18,19,20}$, evidenciando o potencial da Teleodontologia de tornar o atendimento à população mais acessível.

Em um estudo, foi observado que a prática da higiene dental assistida por Teleodontologia descrita no trabalho, apresentou um modelo de prática de nível médio que pode fornecer cuidados de saúde bucal preventivos abrangentes e serviços de diagnóstico para a crescente população de pacientes subatendidos em áreas urbanas e áreas remotas ${ }^{18}$. Na África do Sul, um estudo constatou ser a Teleodontologia uma solução potencial mais acessível na realização de serviços odontológicos na maioria das áreas rurais da África do $\mathrm{Sul}^{19}$. Já nos Estados Unidos observou-se haver uma demanda crescente para o modelo de casa odontológica virtual, sendo reconhecida e comunicada entre aqueles interessados em acesso a cuidados odontológicos e saúde bucal para as populações vulneráveis e desatendidas ${ }^{20}$.

Em outro trabalho, observou-se que, dentistas generalistas recém-formados, quando supervisionados remotamente, podem fornecer overdentures (prótese total apoiada em implantes) de qualidade satisfatória para a população rural. Esta estratégia tem o potencial para melhorar o acesso aos cuidados e elevar o nível de odontologia disponível para a população rural, quando o encaminhamento para especialistas não é viável ${ }^{21}$.

Revisão de literatura que avaliou a utilização da internet banda larga, telefonia móvel, fotografia digital e websites, constatou que o uso de tecnologias já acessíveis no mercado pode viabilizar os serviços de Teleortodontia no Brasil, como ferramentas de auxílio à prática clínica e à educação continuada $^{22}$. O planejamento, diagnóstico e consulta por meio da Teleodontologia e o uso dessa tecnologia por estudantes também são alternativas que podem ser de grande valia. Nesse sentido, alguns autores abordaram em seus estudos o diagnóstico clínico e a precisão de consultas pelo serviço de Teleodontologia ${ }^{23,24}$.

Em um trabalho, foram avaliados $\mathrm{e}$ comparados os métodos de detecção visíveis e de infravermelho próximo para a identificação da desmineralização oclusal precoce não cavitada em um modelo de dente extraído ex vivo ${ }^{23}$. Os autores constataram que, embora o Midwest Caries ID ${ }^{\mathrm{TM}}$ (MID) e a Tomografia de Coerência Óptica de Polarização Cruzada (CP-OCT) 
tenham sido úteis na detecção da presença de desmineralização, porém não foi possível avaliar adequadamente a profundidade da desmineralização. Assim, o MID e CP-OCT não tinham valores diagnósticos marcadamente superiores a partir da avaliação do exame fotográfico visual (CAM) simples para uso em Teleodontologia.

Em um outro estudo, foi constatado que o uso da tecnologia da informação pode aumentar a precisão de consultas em medicação oral, sendo aumentada, com a participação de dois especialistas remotos, a possibilidade de diagnóstico correto ${ }^{24}$.

Em estudo realizado no Reino Unido objetivou-se avaliar se o planejamento do tratamento ortodôntico é reprodutível quando realizado usando registros digitais em comparação com exames clínicos ou uso de registros padrão, bem como a opinião dos pacientes sobre consultas presenciais e uso potencial da Teleodontologia. Foi constatado que a reprodutibilidade do planejamento do tratamento intra-observador foi influenciada por uma mudança no formato de informação diagnóstica para metade dos observadores; o acordo de planejamento de tratamento entre observadores foi influenciado por informações de diagnóstico e que nenhum indivíduo estava insatisfeito com o sistema de consulta convencional $^{25}$.

Um estudo nos Estados Unidos com estudantes de pós-graduação em Odontologia, demonstrou que tais discentes preferiram os formatos presenciais e assíncronos em relação ao formato síncrono, em termos de eficácia e clareza das apresentações. Assim, o formato assíncrono pode ser uma maneira mais eficaz de ensinar num curso de pós-graduação ${ }^{26}$. Tal resultado ressalta a eficácia do ensino à distância, como publicado em trabalho da literatura ${ }^{27}$. Já na Índia, um trabalho observou que a maioria dos internos e pós-graduandos de Odontologia não dispunham de conhecimento e conscientização adequados sobre a Teleodontologia ${ }^{28}$.

O mais recente estudo selecionado na composição dessa revisão integrativa explorou o impacto potencial de um sistema de telemedicina oral móvel na especialidade de saúde bucal. Os autores sugerem que a Teleodontologia pode otimizar o uso de hipóteses diagnósticas e habilidades de especialistas, em regiões remotas, onde os dentistas são escassos ${ }^{29}$.

\section{DISCUSSÃO}

A quantidade de artigos selecionados, apesar do grande número de artigos encontrados após as combinações dos descritores definidos, pode ser explicada por não ser incluída na busca dos artigos a literatura cinzenta, além do termo "Teleodontologia" não ser um descritor cadastrado nos Descritores em Ciências da Saúde $(\mathrm{DeCS})^{30}$. Ainda, nas bases de dados em que foram feitas as buscas para esta revisão, muitas revistas da área de educação não estão indexadas, onde muitos artigos sobre a temática Teleodontologia podem estar publicados, tendo uma maior indexação de revistas da área da saúde.

Muitos estudos e publicações sobre o avanço das Tecnologias de Informação e Comunicação (TIC) são oriundos de países desenvolvidos, como os Estados Unidos, pois além de tecnologia avançada, são necessários recursos humanos treinados e capacitados para um adequado e exitoso serviço de Telessaúde ${ }^{10}$.

Os dois primeiros artigos elencados para essa revisão tratam da Teleodontologia como uma nova era na Odontologia. Isso pode ser explicado pela constante evolução dos serviços de Teleodontologia, em especial quanto à Teleassistência e Teleducação ${ }^{6}$. Como exemplo de uma experiência exitosa da Teleodontologia, tem-se o Núcleo de Teleodontologia da 
Faculdade de Odontologia da Universidade de São Paulo que, vinculado ao Programa Telessaúde Brasil, onde desde 2007 atende às demandas da faculdade e da comunidade por meio da Teleducação e Teleassistência, respectivamente ${ }^{31}$.

Alguns artigos dessa revisão integrativa abordaram o uso da Teleodontologia na temática da Teleassistência, ofertando um maior acesso aos serviços de assistência odontológica, minimizando, assim, as barreiras geográficas entre profissionais e comunidades ${ }^{18-22}$. Isso se dá por muitos cirurgiões-dentistas estarem concentrados nos grandes centros, havendo uma distribuição desigual destes profissionais, considerando as diversas regiões brasileiras. Tal fator pode contribuir na dificuldade de acesso da população brasileira aos serviços de saúde bucal $^{32}$

Outros estudos selecionados para compor essa revisão tratam da Teleodontologia como ferramenta da Teleducação ${ }^{22-29}$, podendo ser usada na formação profissional desde a graduação até a pós-graduação. Assim, a Teleodontologia pode ser considerada excelente forma de integrar tecnologias e educação, corroborando com trabalho já publicado na literatura $^{08}$.

Em estudo publicado, os autores afirmaram que há muitas maneiras criativas de agregar a tecnologia ao dia-a-dia, estando a Teleodontologia oferecendo ao clínico/ especialista maneiras inovadoras de agregar valor à prática diária ${ }^{7}$.

\section{CONCLUSÃO}

Os estudos selecionados para essa revisão integrativa mostram que a Teleodontologia é uma realidade global, sendo uma ferramenta importante para o auxílio da assistência odontológica, em especial às populações mais vulneráveis e formação continuada do cirurgião- dentista.

É preciso que mais estudos sejam realizados mostrando a efetividade dessa tecnologia. Além disso, os resultados das experiências do uso dessa tecnologia necessitam serem difundidos e disseminados para a comunidade científica em geral, inclusive nas bases de dados em saúde. Isso possibilitará a ampliação e consolidação desses serviços no mundo, preenchendo as lacunas ainda existentes quanto à sua evidência, possibilitando um maior embasamento para sua ampliação e consolidação.

\section{ABSTRACT Situational panorama of Teleodontology in the world: an integrative review}

The use of Information and Communication Technologies (ICT) for the exchange of data and information in Dentistry, providing health services in situations where it is necessary to overcome geographical, temporal, social and cultural barriers is called Teleodontology. The objective of this integrative review was to analyze the overall situational panorama of Teleodontology. Scientific articles published in the last 10 years have been selected, in full, in portuguese and in english. The search for the journals was carried out in the Scientific Eletronic Library Online databases, LILACS and PubMed, by means of combining the following descriptors: "Telemedicine", "Dentistry", "Education, Distance", in portuguese and english. A total of 340 articles were found, of which 14 were included for final evaluation, with PubMed the most prevalent database $(71.4 \%)$. Most studies $(85.7 \%)$ were included in level 4 evidence. Were identified studies reporting the use of this technology to minimize geographical barriers and to provide adequate assistance to the most vulnerable populations and others on reports from professionals, dentists and patients regarding the use of Teleodontology. It was concluded that Teleodontology is a global reality, but still needs to disseminate its experiences to 
the scientific community, for its consolidation as an educational and health care tool.

Descriptors: Information Technology. Telemedicine. Dental Informatics. Dentistry. Education, Distance.

\section{REFERÊNCIAS}

1. Haddad AE, Skelton-Macedo MC. Teleodontologia na formação dos profissionais de saúde. In: Mathias I, Monteiro A. (Org.). Gold book: Inovação tecnológica em educação e saúde. Rio de Janeiro: EdUERJ; 2012 [Acesso em 25 maio 2017]. Disponível em: http:// www.telessaude.uerj.br/resource/goldboo k/pdf/12.pdf 11.

2. Folke LE. Teledentistry. An overview. Tex Dent J 2001; 118:10.

3. Nuttall, N. M. The Highlands and Islands Teledentistry Project. Final Report to NHS R\&D, 2002.

4. Bradley M, Black P, Noble S, Thompson R, Lamey PJ. Application of teledentistry in oral medicine in a Community Dental Service, N Ireland BDJ. 2010; 209(8):399-404.

5. Brasil. Departamento de Atenção Básica (DAB). Telessaúde. Telessaúde Brasil Redes na Atenção Básica à Saúde. [Acesso em 17 out. 2017]. Disponível em: http://dab.saude.gov.br/portaldab/ape teless aude.php.

6. Cartes-Velasquez R, Bustos-Leal A. Teleodontología: Conceptos, experiencias y proyecciones. Odontoestomatología. 2012; 14(20):17-25.

7. Skelton-Macedo $\mathrm{MC}$, Jacob $\mathrm{CH}$, Ramos DLP, Cardoso RJA, Antoniazzi JH. Teleodontologia: valores agregados para o clínico/especialista. Rev Assoc Paul Cir Dent 2012; 66(2):95-9.

8. Caldarelli PG, Haddad AE. Teleodontologia em consonância com as Diretrizes
Curriculares Nacionais no desenvolvimento de competências profissionais. Rev ABENO. 2016; 16 (2): 25-32.

9. Haddad AE, Skelton-Macedo MC, Andrade RP, Braga WS, Campos FE. Telessaúde. Brasil: ampliando a resolubilidade em Atenção Primária. In: XII Congresso Brasileiro de Informática em Saúde, 2010, Porto de Galinhas, PE. Anais do XII Congresso Brasileiro de Informática em Saúde, 2010.

10. Haddad AE, Skelton-Macedo MC. Teleodontologia na formação dos profissionais de saúde. Gold Book, 2014; 173-206.

11. Boringi $\mathrm{M}$, Waghray S, Lavanya $\mathrm{R}, \mathrm{Babu}$ DB, Badam RK, Harsha N, et al. Knowledge and Awareness of Teledentistry among Dental Professionals - A Cross Sectional Study. J Clin Diagn Res. 2015; 9(8):ZC41-4.

12. Cartes-Velásquez R, Bustos-Leal A. Teleodontología: Conceptos, experiencias y proyecciones. Odontoestomatología. 2012; 14(20):17-25.

13. Souza MT, Silva MD, Carvalho R. Integrative review: what is it? How to do it? Einstein 2010; 8(1 Pt 1):102-6.

14. Stetler CB, Brunell M, Giuliano KK, Morsi D, Prince L, Newell-Stokes V. Evidencebased practice and the role of nursing leadership. J Nurs Adm. 1999; 28(7-8):45-53.

15. Stetler CB, Morsi D, Rucki S, Broughton S, Corrigan B, Fitzgerald J, et al. Utilizationfocused integrative reviews in a nursing service. Appl Nurs Res. 1998; 11(4):195-206.

16. Jampani ND, Nutalapati R, Dontula BSK, Boyapati R. Applications of teledentistry: A literature review and update. J Int Soc Prev Community Dent. 2011 Jul-Dec; 1(2):37-44.

17. Silva ASC, Rizzante FAP, Picolini MM, Capos K, Corrêa CC, Franco EC, et al. Bauru School of Dentistry Tele-Health League: an 
educational strategy applied to research, teaching and extension among applications in tele-health. J Appl Oral Sci. 2011; 19(6):599603.

18. Summerfelt FF. Teledentistry-Assisted, Affiliated Practice for Dental Hygienists: An Innovative Oral Health Workforce Model. J Dent Educ. 2011; 75(6):733-42.

19. Fortuin JB, Naidoo S. Opportunities for Teledentistry in South Africa. SADJ. 2015; 70(8):342-46.

20. Glassman P, Harrington M, Mertz E, Namakian M. The Virtual Dental Home: Implications for Policy and Strategy. J Calif Dent Assoc. 2012; 40(7):605-11.

21. Keeppanasserril A, Matthew A, Muddappa S. Effectiveness of Tele-guided Interceptive Prosthodontic treatment in rural India: A comparative pilot study. Online J Public Health Informatics. 2011; 3(2):1-8.

22. Costa ALP, Silva AA, Pereira CB. Teleortodontia: ferramenta de auxílio à prática clínica e à educação continuada. Dental Press J Orthod 2011; 16(6):15-21.

23. Hilsen ZV, Jones, RS. Comparing potential early caries assessment methods for teledentistry. BMC Oral Health. 2013; 13(16):1-9.

24. Torres-Pereira CC, Morosini IAC, Possebon RS, Giovanini AF, Bortoluzzi MC, Leão JC et al. Teledentistry: Distant diagnosis of oral disease using e-mails. Telemed e-Health. 2013; 19(2):117-21.

25. Dunbar AC, Bearn D, McIntyre G. The influence of using digital diagnostic information on orthodontic treatment planning - a pilot study. J Healthcare Eng. 2014; 5(4):411-28.

26. Kunin M, Julliard KN, Rodriguez TE. Comparing Face-to-Face, Synchronous, and Asynchronous Learning: Postgraduate
Dental Resident Preferences. J Dent Educ. 2014; 78(6):856-66.

27. Santos ACZF, Andrade IKR, Piva MR, Takeshita WM. Avaliação e desenvolvimento de ambiente virtual de aprendizagem no ensino da disciplina de Diagnóstico Oral por meio do blended learning. Rev ABENO. 2017; 17(2):76-87.

28. Boringi M, Waghray S, Lavanya R, Babu DBG, Badam RK, Harsha $\mathrm{N}$, et al. Knowledge and awareness of Teledentistry among Dental professionals - a cross sectional study. JCDR. 2015; 9(8):ZC41ZC44.

29. Tesfalul M, Littman-Quinn R, Antwi C, Ndlovu S, Motsepe D, Phuthego M, et al. Evaluating the potential impact of a mobile telemedicine system on coordination of specialty care for patients with complicated oral lesions in Botswana. J Am Med Inform Assoc. 2016; 23:e142-e145.

30. Descritores em Ciências da Saúde: DeCS [Internet]. Ed. 2017. São Paulo (SP): BIREME/ OPAS/ OMS. 2017 [atualizado 2017 Mai; Acesso em 17 out. 2017]. Disponível em: http://decs.bvsalud.org.

31. Brasil. Universidade de São Paulo. Faculdade de Odontologia. Núcleo de Teleodontologia. [Acesso em 17 out. 2017]. Disponível em: http://www.fo.usp.br/?page_id=2068.

32. Correia DL, Jorge RR. Distribuição dos cirurgiões-dentistas no brasil e o acesso da população aos serviços de saúde bucal. Programa de Iniciação Científica UNIGRANRIO; 2013.

\section{Correspondência para:}

Maria Imaculada de Queiroz Rodrigues e-mail: imaculadaqueirozr1997@gmail.com Rua Guilherme Perdigão, 240 Parangaba 60720-420 Fortaleza/CE 